\title{
FOOT TYPE CLASSIFICATION FOR CHINESE CHILDREN AND ADOLESCENTS
}

\author{
Miaomiao Xu${ }^{1,2}$, Jing Xian $\mathrm{Li}^{3}$, Youlian Hong ${ }^{4}$, and Lin Wang, \\ ${ }^{1}$ Key Laboratory of Exercise and Health Science of the Ministry of Education, Shanghai \\ University of Sports, Shanghai, China \\ ${ }^{2}$ School of Kinesiology, Shanghai University of Sports, Shanghai, China \\ ${ }^{3}$ School of Human Kinetics, University of Ottawa, Ottawa, Ontario, Canada \\ ${ }^{4}$ Department of Sports Medicine, Chengdu Sports University, Chengdu, Sichuan, China
}

Original scientific paper

https://doi.org/10.26582/k.51.1.8

\begin{abstract}
:
The objective of this study was to examine the three-dimensional foot shape data and determine foot type's distribution among Chinese children and adolescents. A total of sixteen three-dimensional foot shape variables of 5,069 Chinese children were measured through filming, including 3 girth-related variables, 3 lengthrelated variables, 2 width-related variables, and 8 height-related variables. Cluster analysis was performed to classify these three-dimensional feet data of Chinese children and adolescents into three identified foot types, namely Robust Feet, Slender Feet, and Flat Feet, which differed in terms of length, volume, and arch height. The distribution of the three foot types varied across the different foot length groups. The foot types classification may be used in the design of shoe lasts and in the comfortable footwear manufacturing to minimize error fitting.
\end{abstract}

Key words: foot shape, cluster analysis, shoes making

\section{Introduction}

Human foot has an irregular three-dimensional (3D) shape. Information about important foot characteristics helps not only to improve shoe comfort (Price \& Nester, 2016), but also to maintain the proper physiological development of the feet (Barisch-Fritz, Schmeltzpfenning, Plank, \& Grau, 2014). Currently, shoes for children are designed by linearly scaling molds taken from adult feet. However, the proportions of children's feet do not correspond to those of adults (Mauch, Grau, Krauss, Maiwald, \& Horstmann, 2009), consequently, children footwear may not be optimally adapted. In view of the differences in the foot morphologies of children, the foot dimensions of children should be collected, and their foot shape characteristics should be analyzed (Lee \& Wang, 2015).

Previous studies used 3D scanning in their methodology and analyzed the reduced morphological measurements of the feet by using cluster analysis (Mauch, et al., 2009; Mauch, Grau, Krauss, Maiwald, \& Horstmann, 2008). For example, in the study conducted by Mauch et al. (2009), 3D foot morphology was analyzed with a sample of 2,869 children aged between 2 and 14 years, and a cluster analysis was used to present three different foot types between boys and girls. However, the said study involved a German sample, and its results cannot be extrapolated to a Chinese sample.

Currently, 3D data on the feet of Chinese children and adolescents are still lacking, and statistical multivariate methods have not yet been used to cluster similar feet into groups. Therefore, the purpose of this study was to examine the differences among the feet of Chinese children and adolescents aged 7-18 years by clustering them into groups. The following hypotheses were considered in this study: (1) feet can be classified as Robust Feet, Slender Feet and Flat Feet; (2) the foot types distribution varies across the different foot length groups of Chinese children and adolescents.

\section{Methods}

\section{Participants}

In this study, a stratified sampling was used to select samples in seven regions in China, respectively North China, Southern China, East China, Central China, Southwest China, Northwest China and Northeast China. In every region, at least thirty 
participants were chosen respective to each gender and age. A total of 2,492 boys and 2,577 girls of school age (7-18 years) from Chinese primary, secondary, and senior high schools volunteered for this study. Their parents/guardians, who were previously informed of the study, signed a letter of consent to confirm their children's participation. Recent injuries in the lower extremities, foot bone disorders, or skin infections were excluding criteria. The study was approved by the local Ethics Committee.

\section{Procedures}

Before the foot shapes of the participants were measured, their heights were measured under barefoot condition using a stadiometer (Harpenden stadiometer, model 98.603, HoltainLtd, Crosswell, UK). Body mass was determined by a digital weighing scale (Seca electronic scale, model 770, Hamburg, Germany). The participants wore light clothing when the measurements were taken.

Multiple image-based approaches were used. The participants' feet were first recorded using four video cameras (SONY HVR-Z1C, Japan). The 3D foot shape data of the right foot were collected using a video filming system. The foot model developed by the Biomechanics Institute of Valencia, Spain (García-Hernández, et al., 2005; Hong, Wang, Xu, $\& \mathrm{Li}, 2011)$ was used in the current study. The model has thirteen anatomical reference points, which were marked with a black marker pen prior to filming. These reference points were defined manually by the same investigator (Figure 1). The participants had to stand still while being filmed, in a bipedal support, with their body weight evenly distributed on both feet. Four digital cameras were used to film the right foot synchronously for at least $5 \mathrm{~s}$. The sample frequency was set to $50 \mathrm{~Hz}$.

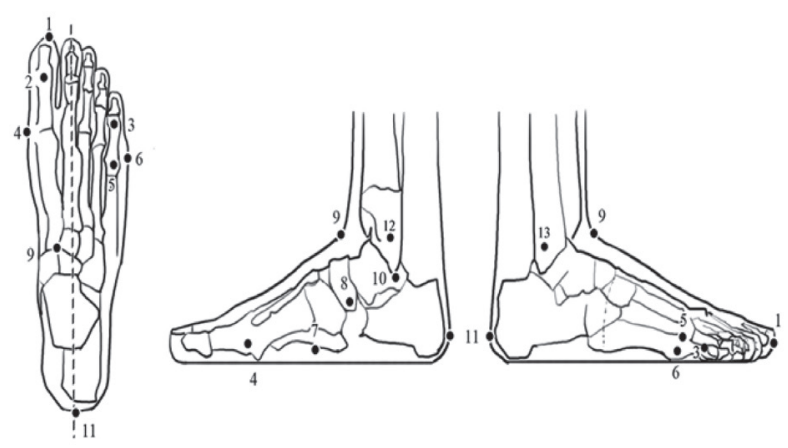

Note. 1 - front end of the longest toe; 2 - first phalangeal head; 3 - fifth phalangeal head; 4 - first metatarsal head; 5 - highest point of the fifth metatarsal head; 6 - fifth metatarsal head; 7 lowest point of the foot arch; 8 - lowest scaphoid point; 9 - point where the leg meets the foot; 10 - the tip of the medial malleolus; 11 - pternion; 12 - medial malleolus; 13 - Lateral malleolus

Figure 1. Reference landmarks.
Three girth variables, namely, ball girth (BG), midfoot girth (MFG), and malleolar girth (MG), were measured to the nearest $1 \mathrm{~mm}$ using a flexible tape (Figure 2). The girths were measured by the same investigator who defined the reference points.

After filming, the coordinates of the thirteen reference points were obtained by the automatic digitization of the video image by Ariel Motion Analysis System (Ariel Dynamics, USA). The digitized data were then smoothed using a Butterworth filter at $6 \mathrm{~Hz}$.

\section{Variables}

The measurements shown in Figure 2 were obtained from the coordinates of the thirteen reference points. The number of variables analyzed in this study exceeded the number of variables used in several previous studies (Mauch, et al., 2008, 2009).

The three variables related to length were foot length (FL), medial ball length (MBL), and forefoot length (ForeL). The two variables related to width were foot width (FW) and heel width (HW). The eight variables related to height included the first phalangeal head height $(\mathrm{P} 1 \mathrm{H})$, the fifth phalangeal head height $(\mathrm{P} 5 \mathrm{H})$, the fifth metatarsal head height (M5H), arch height (ArH), the lowest scaphoid point height (LSH), instep height (IH), medial malleolus height $(\mathrm{MMH})$, and calcaneus height $(\mathrm{CH})$. Thus, together with the three girth variables measured before the filming, a total of 16-foot shape variables were measured. This method was used to measure the three-dimensional foot variables in previous studies (Li, Xu, Wang, \& Shao, 2005; Hong, et al., 2011). A high reliability of the measurements was found, with the interclass correlation coefficients ranging from 0.930 to 0.999 (Hong, et al., 2011).

\section{Statistical analysis}

Statistical analysis was performed using the SPSS 20.0 software package, with a significance level set at $\mathrm{p}<.05$. A principal component analysis (PCA) was then conducted to extract the main feature parameters for describing the foot shape differences. This method was used to develop foot type classification schemes in previous studies (Lee \& Wang, 2015; Mauch, et al., 2008). Ward's hierarchical method was used to determine the number of clusters. The results were optimized by $K$-means clustering, with the cluster centers from the previous analysis as the initial seed points.

\section{Results}

\section{Participants' physical characteristics}

Physical characteristics of the participants are summarized in Table 1. 

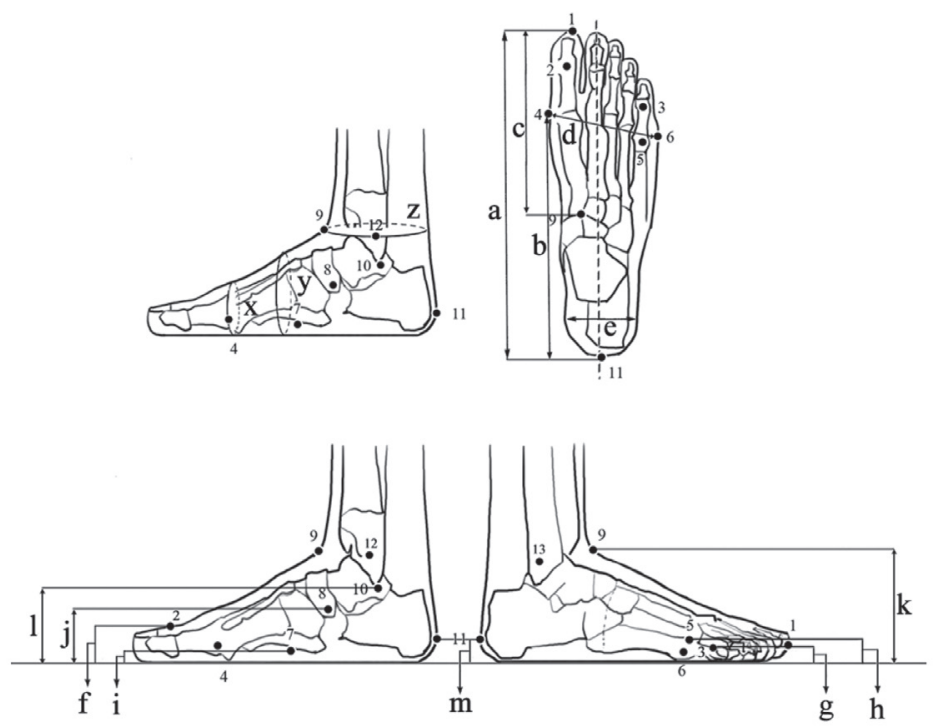

Note. 3 - length-related variables: $a$ - foot length ( $F L$ ); $b$ - medial ball length (MBL); $c$ - forefoot length (ForeL). 2 - width-related variables: $d$ - foot width (FW); e - heel width (HW). 8 - height-related variables: $f$ - height of the first toe (T1H ); $g$ - height of the fifth toe (T5H); $\mathrm{h}$ - height of the metatarsal head (M5H); i - arch height(ArH); j - lowest scaphoid point height (LSH); $\mathrm{k}$ - instep height $(\mathrm{IH}) ; \mathrm{I}$ - medial malleolus height (MMH); $\mathrm{m}$ - calcaneus height $(\mathrm{CH})$. 3 - girth-related variables: $\mathrm{x}-$ ball girth (BG); $y-$ midfoot girth (MFG); z - malleoli girth (MG).

Figure 2. Foot measurements.

Table 1. Physical characteristics of the participants are summarized in the study. Values are means(SD)

\begin{tabular}{|c|c|c|c|c|}
\hline Age (years) & Gender & Height $(\mathrm{cm})$ & Weight (kg) & Foot length $(\mathrm{mm})$ \\
\hline \multirow[t]{2}{*}{7} & Boys $(n=276)$ & $126.3(4.5)$ & $27.7(4.6)$ & $195.1(9.4)$ \\
\hline & Girls $(n=283)$ & $125.0(4.9)$ & $25.4(3.8)$ & $190.2(8.7)$ \\
\hline \multirow[t]{2}{*}{8} & Boys $(n=218)$ & $131.8(5.9)$ & $30.8(7.0)$ & 203.5(12.4) \\
\hline & Girls $(n=215)$ & $130.4(5.7)$ & $28.3(5.2)$ & $200.3(11.0)$ \\
\hline \multirow[t]{2}{*}{9} & Boys $(n=187)$ & $138.9(6.8)$ & $37.9(10.5)$ & $214.9(13.8)$ \\
\hline & Girls $(n=183)$ & $138.2(6.5)$ & $33.1(7.1)$ & $210.7(12.1)$ \\
\hline \multirow[t]{2}{*}{10} & Boys $(n=232)$ & $142.3(6.5)$ & $37.7(8.5)$ & $220.0(12.1)$ \\
\hline & Girls $(n=232)$ & $142.0(7.1)$ & $35.9(8.6)$ & $216.0(11.9)$ \\
\hline \multirow[t]{2}{*}{11} & Boys $(n=176)$ & $150.2(7.9)$ & $46.5(11.1)$ & 233.3(13.9) \\
\hline & Girls $(n=190)$ & $150.8(7.3)$ & $42.4(9.0)$ & $226.0(12.0)$ \\
\hline \multirow[t]{2}{*}{12} & Boys $(n=151)$ & $156.0(8.1)$ & $51.4(11.8)$ & $240.5(13.2)$ \\
\hline & Girls $(n=200)$ & $154.5(5.9)$ & $47.9(10.1)$ & 229.6(9.4) \\
\hline \multirow[t]{2}{*}{13} & Boys $(n=224)$ & $160.1(7.9)$ & $51.5(11.6)$ & $246.0(12.7)$ \\
\hline & Girls $(n=208)$ & $156.8(6.1)$ & $48.8(9.1)$ & $230.3(9.4)$ \\
\hline \multirow[t]{2}{*}{14} & Boys $(n=227)$ & $165.0(8.5)$ & $56.6(13.2)$ & $249.9(13.3)$ \\
\hline & Girls $(n=256)$ & $160.1(5.9)$ & $51.7(8.1)$ & 232.3(9.3) \\
\hline \multirow[t]{2}{*}{15} & Boys $(n=174)$ & $169.9(7.3)$ & 61.1(12.2) & $252.0(11.8)$ \\
\hline & Girls $(n=193)$ & $162.5(6.3)$ & $56.5(8.8)$ & $236.6(10.1)$ \\
\hline \multirow[t]{2}{*}{16} & Boys $(n=189)$ & $172.3(6.3)$ & $63.8(10.6)$ & $253.3(10.6)$ \\
\hline & Girls $(n=182)$ & $160.9(5.6)$ & $56.1(7.1)$ & $232.3(10.4)$ \\
\hline \multirow[t]{2}{*}{17} & Boys $(n=238)$ & $172.5(6.9)$ & $64.2(11.9)$ & $252.5(11.6)$ \\
\hline & Girls $(n=237)$ & $161.8(6.3)$ & $56.9(8.1)$ & 233.3(10.7) \\
\hline \multirow[t]{2}{*}{18} & Boys $(n=200)$ & $173.9(6.2)$ & $64.8(8.7)$ & $253.4(10.8)$ \\
\hline & Girls $(n=198)$ & $162.8(5.4)$ & $57.1(7.8)$ & 232.3(9.3) \\
\hline \multirow[t]{2}{*}{ Total } & Boys $(n=2492)$ & $154.0(18.1)$ & $48.7(16.7)$ & $233.2(24.3)$ \\
\hline & Girls $(n=2577)$ & $149.8(14.6)$ & $44.5(13.9)$ & $221.6(18.3)$ \\
\hline
\end{tabular}




\section{PCA results}

Three principal components (PCs) were obtained and the percentage of the total variance relative to foot length explained by the three principal components was $83.12 \%$. PC 1 was lengthrelated, including FL, MBL, ForeL, $\mathrm{IH}$ and $\mathrm{CH}$. Therefore, PC 1 was named the "length factor". PC 2 was volume-related, including FW, HW, BG, MFG, MG, P1H, P5H, and M5H. Thus, PC 2 was named the "volume factor". The variables involved in PC 3 were arch-related, including ArH, LSH, and $\mathrm{MMH}$, so PC 3 was named the "arch factor" (Table 2).

Table 2. PCA results for 16 three-dimension foot variables relative to foot length

\begin{tabular}{lccc}
\hline & \multicolumn{3}{c}{ Principal components } \\
\cline { 2 - 4 } Variables (\%FL) & PC1 & PC2 & PC3 \\
\hline BG & & 0.64 & \\
MFG & & 0.65 & \\
MG & & 0.65 & \\
FL & 0.73 & & \\
MBL & 0.7 & & \\
ForeL & 0.81 & & \\
FW & 0.64 & 0.63 & \\
HW & & 0.66 & \\
P1H & & 0.62 & \\
P5H & & 0.8 & 0.92 \\
M5H & & 0.82 & 0.62 \\
ArH & & & 0.82 \\
LSH & & & \\
MMH & & & \\
IH & & & \\
CH & 0.73 & & \\
Eigenvalue & 10.87 & & \\
Variance & $31.27 \%$ & $29.86 \%$ & \\
explained (\%) & $31.27 \%$ & $61.14 \%$ & \\
Cumulative (\%) & 0.83 & & \\
\hline & & & \\
\hline
\end{tabular}

Note. BG - ball girth; MFG - midfoot girth; MG - malleolar girth; $\mathrm{FL}$ - foot length; $\mathrm{MBL}$ - medial ball length; ForeL forefoot length; FW - foot width; HW - heel width; P1H - first phalangeal head height; $\mathrm{P} 5 \mathrm{H}$ - fifth phalangeal head height; $\mathrm{M} 5 \mathrm{H}$ - fifth metatarsal head height; ArH - arch height; LSH lowest scaphoid point height; $\mathrm{IH}$ - instep height; $\mathrm{MMH}$ - medial malleolus height; $\mathrm{CH}$ - calcaneus height.

\section{Classifying foot shapes}

The standardized and independent factors, namely, length, volume, and arch, were used in the hierarchical analysis. Three clusters that represent different foot types were identified on the basis of the three factors. Profiles of the different foot types were described by their optimized cluster center (QUICK CLUSTER) as follows (Figure 3):

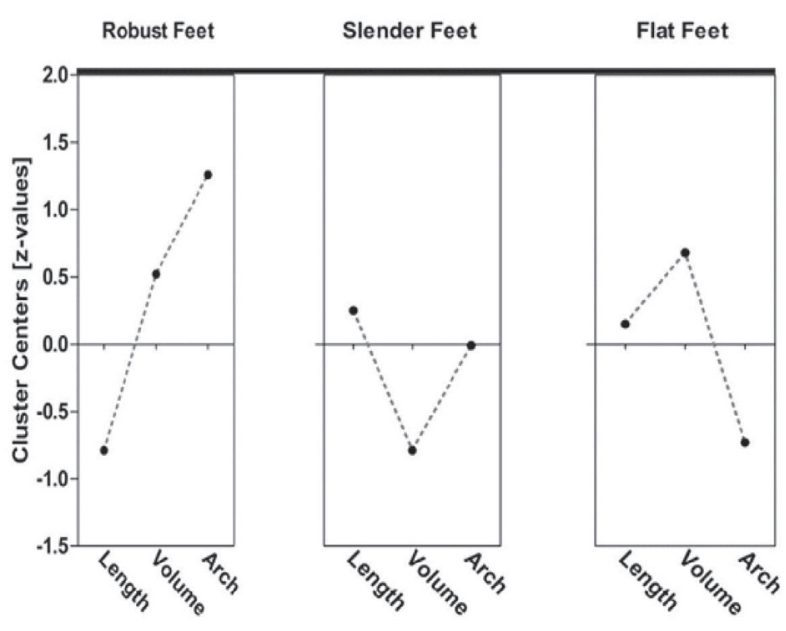

Figure 3. Profiles of three foot types. The $x$ axis shows the three principal components; the y axis shows $z$-values of the cluster centers of each cluster, whereby large positive or negative z-values correspond to an increased or decreased dominance of the factor characteristic within the particular foot clusters, a value around zero represents a medium characteristic.

(1) Robust Feet $(n=1052 ; 21 \%)$ : This foot type was mainly characterized by a fairly high arch and a wide volume. The variables included in the length factor exhibited average $z$-values of approximately -0.79 , indicating a short foot length of this foot type.

(2) Slender Feet $(n=2233 ; 44 \%)$ : The feet in this cluster were characterized by their very small volume, that is, they had narrow ball and heel widths as well as a medium arch height. In addition, they displayed a relatively long foot length.

(3) Flat Feet $(n=1784 ; 35 \%)$ : Compared with the slender feet, this foot type had a relatively large volume and a low arch height. The variables included in the length factor exhibited average $z$-values of approximately 0.15 , indicating a medium foot length of this foot type.

\section{Distributions of the three foot types in the different foot length groups}

The proportions of the foot types in each foot length group totaled $100 \%$. The foot type distribution varied across the different foot length groups. A high proportion of Slender Feet existed in the smaller sizes (foot lengths from $175 \mathrm{~mm}$ to 215 $\mathrm{mm})$. In fact, Slender Feet accounted for more than $50 \%$ of each size up to $215 \mathrm{~mm}$ foot length. By contrast, the Flat Feet type was more represented in the larger sizes (foot lengths from $240 \mathrm{~mm}$ to $280 \mathrm{~mm}$ ); this foot type accounted for over $40 \%$ of each size in this foot length group, except for 260 $\mathrm{mm}$ foot length. An equal mixture of all foot types was found between the foot lengths of $220 \mathrm{~mm}$ and $235 \mathrm{~mm}$ (Figure 4). 


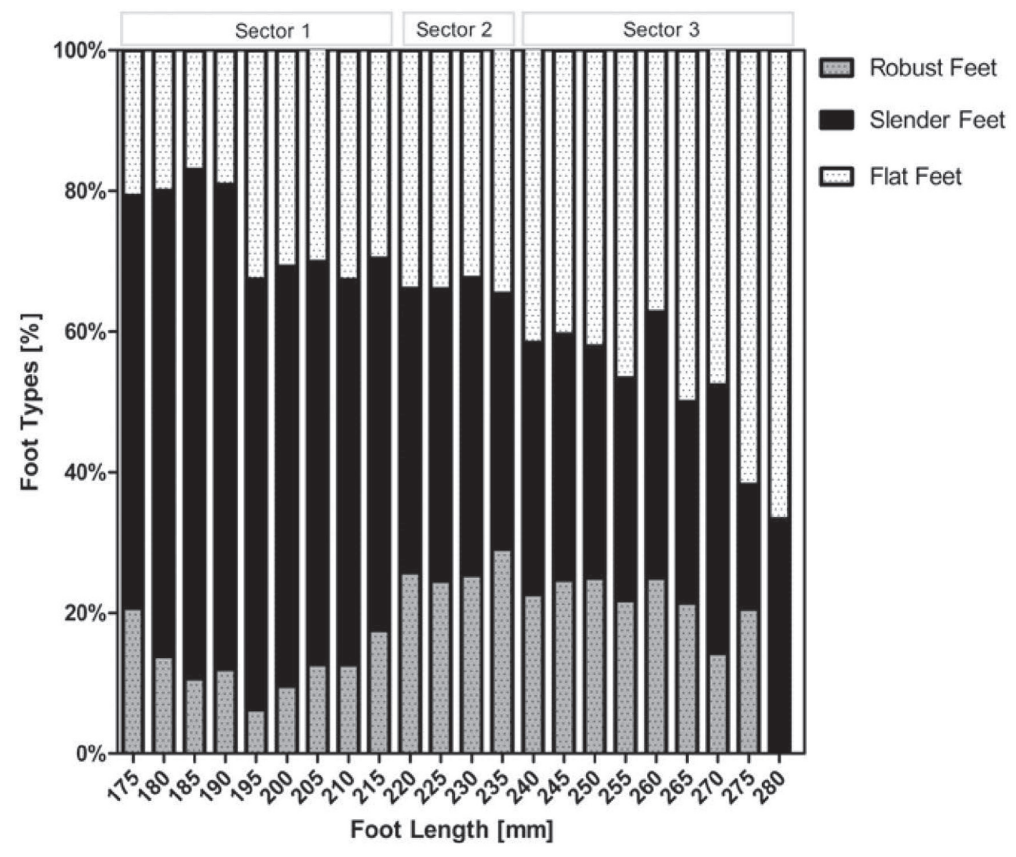

Figure 4. Distribution of the three foot types according to foot length categories.

\section{Discussion and conclusions}

This study examined the 3D foot shape data and determined foot type distribution among Chinese children and adolescents. The three different foot types, namely, Robust Feet, Slender Feet and Flat Feet, were identified among the population of Chinese children and adolescents, and the distribution of the three foot types varied across the different foot length groups.

The results from this study are consistent with findings from previous studies that investigated the 3D foot shape classification in children. Mauch et al. (2008) classified German children's feet into five feet types, including flat, slender, robust, short, and long feet using cluster analysis. A comparison of these five feet types with the three foot types identified in the current study shows that the slender and long feet types of the German children can be fused into the Slender Feet type of Chinese youngsters. Furthermore, the robust and short feet types of German children are similar to the Robust Feet type of Chinese kids, which is characterized by a short foot length and a great arch height. The current study is the first large-scale research that investigated the 3D foot shape data of Chinese children and adolescents.

In the serial footwear production, serial lasts are designed on the basis of a prototype last in a regulated length and width graduation (Krauss,Valiant, Horstmann, \& Grau, 2010). Therefore, the calculated foot types of an entire sample are based on a prototype shoe size, which is either upscaled or downscaled to produce other sizes. However, this procedure disregards the differences in the proportions of foot types across different foot sizes, as demonstrated in the current study. That is, approximately two-thirds of the children with 175-215 $\mathrm{mm}$ foot lengths would require shoes designed for a Slender Feet type. If this subsample (175-215 $\mathrm{mm}$ foot lengths) is considered separately from the entire sample size range (175-280 $\mathrm{mm}$ foot lengths), Robust Feet or Flat Feet might be unaccounted for when using the grand mean of the entire population. Therefore, the foot type distributions across different foot lengths of children and adolescents should be the basis for recommending a shoe design that considers the foot type classification to improve shoe fit.

Mauch et al. (2009) suggested that the distribution of the foot types in the same shoe size could explain the shoe size-related discrepancies among German children. The results of the current study support their view that different foot length sizes have different foot type distributions. However, Mauch et al. (2009) suggested a high proportion of robust feet existed in small shoe sizes (25-28 French size), whereas Slender Feet had a large share in large shoe sizes (35-41 French size) of German children aged 2-14 years. The participants' age range differences and racial differences could explain the foot types distribution differences between the two populations.

A few limitations of the current study should be acknowledged. BMI and physical activity may influence foot shape, which was not considered in the current study. In addition, one important measurement for foot dimension determination is an arch height. The arch height is typically measured by navicular or talonavicular joint line, but that landmark was not digitized in this study. Last, we did not distinguish the flat-foot from the normal-foot in 
the current population. The proportion of the flatfoot may influence the results. These limitations should be considered in further studies.

In conclusion, three different foot types, namely, Robust Feet, Slender Feet and Flat Feet, were identified in this study. The foot type distribu- tion varied across the different foot length groups of Chinese children and adolescents. The results of this study can lay the foundation for the investigation of the development of foot shape and can also provide useful information to consumers and shoe manufacturers.

\section{References}

Barisch-Fritz, B., Schmeltzpfenning, T., Plank, C., \& Grau, S. (2014). Foot deformation during walking: Differences between static and dynamic 3D foot morphology in developing feet. Ergonomics, 57(6), 921-933. doi: 10.1080/00140139.2014.899629

García-Hernández, J., Heras, S., Juan, A., Paredes, R., Nácher, B., Alemany, S., et al. (2005). The MORFO3D Foot Database. Lecture Notes in Computer Science, 3523, 658-665. doi: 10.1007/11492542_80

Hong, Y., Wang, L., Xu, D.Q., \& Li, J.X. (2011). Gender differences in foot shape: A study of Chinese young adults. Sports Biomechanics, 10(2), 85-97. doi: 10.1080/14763141.2011.569567

Krauss, I., Valiant, G., Horstmann, T., \& Grau, S. (2010). Comparison of female foot morphology and last design in athletic footwear-Are men's lasts appropriate for women? Research in Sports Medicine, 18(2), 140-156. doi: $10.1080 / 15438621003627216$

Lee, Y.C., \& Wang, M.J. (2015). Taiwanese adult foot shape classification using 3D scanning data. Ergonomics, 58(3), 513-523. doi: 10.1080/00140139.2014.974683

Li, J.X., Xu, D.Q., Wang, A., \& Shao, K. (2005). Analysis of foot shape measures from Chinese male and female adults. In J. Hamill, E. Hardin \& K. Williams (Eds.), Proceedings of the 7th Symposium on Footwear Biomechanics (pp. 84-86). Brentwood: Footwear Biomechanics.

Mauch, M., Grau, S., Krauss, I., Maiwald, C., \& Horstmann, T. (2008). Foot morphology of normal, underweight and overweight children. International Journal of Obesity, 32(7), 1068-1075. doi: 10.1038/ijo.2008.52

Mauch, M., Grau, S., Krauss, I., Maiwald, C., \& Horstmann, T. (2009). A new approach to children's footwear based on foot type classification. Ergonomics, 52(8), 999-1008. doi: 10.1080/00140130902803549

Mauch, M., Mickle, K.J., Munro, B.J., Dowling, A.M., Grau, S., \& Steele, J.R. (2008). Do the feet of German and Australian children differ in structure? Implications for children's shoe design. Ergonomics, 51(4), $527-539$. doi: 10.1080/00140130701660520

Price, C., \& Nester, C. (2016). Foot dimensions and morphology in healthy weight, overweight and obese males. Clinical Biomechanics, 37, 125-130. doi: 10.1016/j.clinbiomech.2016.07.003

Submitted: February 2, 2018

Accepted: October 10, 2018

Correspondence to:

Lin Wang, MB, PhD

School of Kinesiology, Shanghai University of Sport, China

Tel.: (86) 2151253426

Fax: (86) 2151253470

E-mail: wanglin@sus.edu.cn 